\title{
OBSERVATIONS OF COIMET KOHOUTEK (1973f) WITH A GROUND-BASED FABRY-PEROT SPECTROMETER
}

\author{
D. Huppler, R. J. Reynolds, F.L. Roesler, F Scherb and J. Trauger
}

Between 1973 December 1 and 1974 February 2, optical emission lines from the gas cloud surrounding comet Kohoutek were observed using a double FabryPerot etalon spectrometer at Kitt Peak National Observatory. The spectrometer had a resolving power of 40,000, corresponding to a velocity resolution of about $7.5 \mathrm{~km} \mathrm{sec}^{-1}$. With this resolution it was possible to use the cometearth relative velocity to resolve faint cometary $H_{\alpha} \lambda 6563,[0 I] \lambda 6300$ and other emission lines from geocoronal and airglow emissions and to study the cometary line profiles in order to obtain information about the composition, effective temperatures, outflow velocities, and production rates of atoms and ions in the cometary envelope.

The spectrometer was coupled to the Mcllath Solar Telescope by focussing the primary image of the sky, which has a scale of about 1 arc min per $25 \mathrm{~mm}$, directly onto the $150 \mathrm{~mm}$ diameter etalon of the Fabry-Perot spectrometer Masks could readily be placed just above the Fabry-Perot to restrict the field of view from 5.7 arc minutes down to less than one arc minute as desired. The light passed by the $150 \mathrm{~mm}$ Fabry-Perot was coupled by a 3:1 ratio afocal lens system to a lower resolution $(\delta \lambda \simeq 1.5 \AA) 50 \mathrm{~mm}$ Fabry-Perot which was placed in series with any one of several $50 \mathrm{~mm}$ aperture interference filters with bandpasses typically $15-20 \AA$. The low resolution Fabry-Perot was used to suppress all but one of the narrow $(\delta \lambda \simeq 0.17 \AA)$ transmission peaks of the large FabryPerot which fell within the passband of the interference filter. The FabryPerot etalons were housed in separate gas-tight chambers and pressure-scanned 
over spectral intervals up to $6 \stackrel{\circ}{6}$ using $S_{6}$ as the scanning gas. An automatic pressure difference control system maintained the tune of the two Fabry-Perot etalons during scans. In order to monitor changes in sky brightness and atmospheric transmittance, about $4 \%$ of the light incident on the spectrometer was directed to a reference system containing a $100 \AA$ wide filter centered near the wavelength being scanned. The number of photons counted by the spectrometer and reference system during small equal wavelength scan intervals were punched on paper tape on command from an interferometric refractometer scanned in unison with the Fabry-Perot etalons. This method permitted the direct comparison and addition of scans for enhancing the signal-to-noise ratio.

Analyses of $\mathrm{H} \alpha$ line profiles and line intensities indicate that the mean outflow velocity of the hydrogen atoms was $7.8 \simeq 0.2 \mathrm{~km} \mathrm{~s}^{-1}$ and that the hydrogen atom production rate varied from about $1.0 \times 10^{29} \mathrm{~s}^{-1}$ to about $3.5 \times$ $10^{29} \mathrm{~s}^{-1}$ for comet-sun distances between $1 \mathrm{AU}$ and $04 \mathrm{AU}$, respectively The identification of an $\mathrm{H}_{2} \mathrm{O}^{+}$emission feature in certain Ha scans indicates that the $\mathrm{H}_{2} \mathrm{O}^{+}$ions were moving in a tailward direction with a velocity of 20 to $40 \mathrm{~km} \mathrm{~s}^{-1}$ with respect to the comet nucleus. An upper limit of 1 part in 100 was found for the $\mathrm{D} / \mathrm{H}$ ratio in the cometary atomic hydrogen cloud.

We would like to thank F. Barmore and B. Donn for their assistance and advice.

This work was carried out with support from kitt Peak ivational Observatory, the University of Wiscons in Graduate School, the Planetary Astronomy Program of the National Aeronautics and Space Administration through grant NGR 50-002-242, and the Aeronomy section of the National Science Foundation through grant GA40146.

For complete text see Ap. J. 202, 276, 1975 


\section{DISCUSSION}

W. Jackson: In your slide on the oxygen ${ }^{1} \mathrm{D}$ you estimate $2 \times 10^{28}$ as the production of $\mathrm{O}{ }^{1} \mathrm{D}$ ?

D. H. Huppler: On December 8, from a scan of oxygen with the two arc minute field of view, we estimate $2 \times 10^{28}$, singlet D oxygen atoms per second produced.

W. Jackson: Okay,

From that you can go and calculate what the water production rate should have been on that same day?

D. H. Huppler: If you assume that the only source of singlet $\mathrm{D}$ is $\mathrm{H}_{2} \mathrm{O}$, which we are not sure of. If you do that, then there must be the same number of water, right?

\section{W. Jackson: No.}

The branching ratio for dissociation into $\mathrm{O}^{1} \mathrm{D}$ and $\mathrm{H}_{2}$ is roughly only one or two percent of the total dissociation of the $\mathrm{H}_{2} \mathrm{O}$ molecule.

And if you do that, and look at your same number for $\mathrm{H}$ atoms, you get the same amount of $\mathrm{H}$ atoms produced as you get $\mathrm{O}{ }^{1} \mathrm{D}$.

Now either the $O{ }^{\prime} D$ is produced, as you said, from some other source which either has to have a higher production rate for $O{ }^{1} D$ than water or the $\mathrm{H}$ atom concentration has been grossly underestimated.

Right now from your measurements, the only way you could have that much $O{ }^{1} \mathrm{D}$ is if the $O{ }^{1} \mathrm{D}$ is produced by something else other than water, if you are going to believe your $\mathrm{H}$ measurement in terms of absolute measurements.

D. H. Huppler: Right.

Well, we tried to figure out if there was some way of connecting the two. Primary branching, you said was to $\mathrm{OH}$, and what does $\mathrm{OH}$ break up into, if it does break up.

And we could not find enough things to determine if $\mathrm{OH}$ comes to singlet $\mathrm{D}$. And then there is $\mathrm{CO}^{+}, \mathrm{CO}$, and other molecules containing oxygen for which we couldn't find whether they produced ${ }^{1} \mathrm{D}$ or not. 


\section{DISCUSSION (Continued)}

W. Jackson: The $\mathrm{O}_{2}$ does - we know that. And there is some evidence that $\mathrm{CO}_{2}$. can produce $\mathrm{O}^{1} \mathrm{D}$. If that is the case then in a way you have identified the fact that there is a substantial amount of material that could produce $O{ }^{1} D$ other than water. Now the critical thing is how accurate is that $\mathrm{H}$ measurement? If that $\mathrm{H}$ measurement is off by an order of magnitude, and $\mathrm{I}$ think it is because it disagrees with the ultraviolet measurement, wouldn't you get about $10^{29}$ per second? That is just too much $\mathrm{O}^{\mathrm{l}} \mathrm{D}$.

R. Meier: The hydrogen production rates derived from the Balmer alpha observations are only about a factor of 2 lower than those derived from Lyman alpha observations. A solar Lyman beta line with a factor of two less central reversal can account for the difference, since the excitation flux in the wing of the line would be lower requiring a higher production rate by the same factor.

D. H. Huppler: We get $2 \times 10^{28}$ for oxygen, about $3 \times 10^{29}$ for hydrogen.

H. Keller: I have another question, concerning your production rate determination.

Did you take into account in your heliocentric dependence slides the field of view effect, and how large was the field of view?

D. H. Huppler: The intensities were 2-arc minute field of view because that was the majority of the data that we had.

And for the scans that we had taken with a 6-arc minute field of view we corrected them to a 2-arc minute field.

H. Keller: And did you change your field of view with geocentric distance?

D. H. Huppler: Yes. We also made geocentric distance corrections.

H. Keller: For the field of view effect?

D. H. Huppler: Yes. And they're both talking about the same thing, how much of the comet in kilometers you're looking at for a particular observation so they're all normalized to the 2 arc-minute field of view at $1 \mathrm{AU}$. 


\section{DISCUSSION (Continued)}

H. Keller: Yes. What type of model did you use for it? That depends on the scale length of the species you look at.

D. H. Huppler: Well, for the change from a 6 arc-minute field of view to a 2 arc-minute field of view, we had several days in which both types of scans were taken, so it made it easy for other days when only 6 arc-minutes were taken, and we could just develop the number that we then have to correct the 6 arc-minute scans by, and then we made about the same correction for change in heliocentric distance, and since the scans were only between 0.8 and 1.2 AU heliocentric distance, an error in that will not be too great.

H. Keller: An empirical approach.

D. J. Malaise: When you showed the spectra of oxygen, you pointed out that several spectra were displaced by a certain distance in the head. In what direction were they displaced?

D. H. Huppler: You mean the 40 arc-second displacement. We saw nothing to indicate that there was any asymmetry of the head. So I don't know in particular where that scan was, but we looked and saw nothing that would indicate that sunward, tailward, or perpendicular had any asymmetry.

The hydrogen did have the asymmetry only in the tailward direction. If you go perpendicular to the axis - it looked just like the sunward scans.

D. J. Malaise: Yes. Because there was some suspicion that the oxygen line could extend into the tail, which is very funny, but could the $\mathrm{O}^{1} \mathrm{D}$ level be produced by dissociative recombination of some ion, $\mathrm{H}_{2} \mathrm{O}^{+}$, for instance, in which case you could observe the forbidden line to be asymmetric.

D. H. Huppler: It could potentially be there, but would be less than something like 10 percent of our signal, and so if you're looking for asymmetries of that size, we wouldn't have noticed them. 


\section{DISCUSSION (Continued)}

M. Oppenheimer: Did you derive the production rate by saying that every produced oxygen atom leads to the emission of one photon?

D. H. Huppler: Yes. Because except for right in the center - right close to the head, the collision time is greater than 100 seconds. The densities get low enough fast enough that you would not have a collision.

M. Oppenheimer: How far from the head are we talking about were these observations?

D. H. Huppler: 40 arc-seconds is something like 20,000 kilometers for the field of view.

M. Oppenheimer: I just think the quenching - the chemical reaction rates for oxygen ${ }^{1} \mathrm{D}$ are very high, and you have to be very careful about that.

D. H. Huppler: Right. If it hits anything it's gone.

M. Oppenheimer: - and I think that even out at that distance it's possible that you're underestimating the production rate a little bit, depending on what model you use for the neutral densities. Plus the chemical rates are almost gas-kinetic, which means everything just goes. 